\title{
Precise reddening and metallicity of NGC 6752 from FLAMES spectra ${ }^{\star}, \star \star$
}

\author{
R. G. Gratton ${ }^{1}$, A. Bragaglia ${ }^{2}$, E. Carretta ${ }^{2}$, F. De Angeli ${ }^{3}$, S. Lucatello ${ }^{1}$, G. Piotto ${ }^{3}$, and A. Recio Blanco ${ }^{3,4}$ \\ 1 INAF - Osservatorio Astronomico di Padova, Vicolo dell'Osservatorio 5, 35122 Padova, Italy \\ e-mail: gratton@pd.astro.it \\ 2 INAF - Osservatorio Astronomico di Bologna, Via Ranzani 1, 40127 Bologna, Italy \\ 3 Dipartimento di Astronomia, Universitá di Padova, Vicolo dell'Osservatorio 5, 35122 Padova, Italy \\ ${ }^{4}$ Observatoire Astronomique de la Côte d'Azur, Boulevard de l'Observatoire, BP 4229, 06304 Nice Cedex 4, France
}

Received 8 March 2005 / Accepted 4 May 2005

\begin{abstract}
Accurate reddenings for Globular Clusters could be obtained by comparing the colour-temperature relation obtained using temperatures from a reddening-free indicator $(\mathrm{H} \alpha)$ with that given by standard colour-temperature calibrations. The main difficulty in such derivations is the large errors in temperatures for individual stars due to uncertainties on the removal of instrumental signature for each individual star. The large multiplexing opportunity offered by FLAMES at VLT2 allowed us to obtain spectra centred on $\mathrm{H} \alpha$ at a resolution of $R=6000$ and $5<S / N<50$ for 120 stars near the turn-off of NGC 6752 with GIRAFFE from a single 1300 seconds exposure. This set of spectra was used to derive effective temperatures from fittings of $\mathrm{H} \alpha$ profiles with typical errors of about $\pm 200 \mathrm{~K}$ and reddening estimates with individual errors of $0.05 \mathrm{mag}$. Averaging all individual reddenings, a high precision reddening estimate was obtained for the cluster: $E(B-V)=0.046 \pm 0.005$. The same exposure provided UVES spectra of seven stars near the red giant branch bump at a resolution of 40000 and $20<S / N<40$. These spectra, combined with temperatures from colours (corrected for our high precision reddening value), provided Fe abundances with internal errors of $0.026 \mathrm{dex}$ and with average metallicity $[\mathrm{Fe} / \mathrm{H}]=-1.48 \pm 0.01 \pm 0.06 \mathrm{dex}$ (random + systematic). Abundances were obtained for several other elements, allowing e.g. an accurate estimate of the ratio between the $\alpha$-elements and $\mathrm{Fe}([\alpha / \mathrm{Fe}]=+0.27 \pm 0.01)$. The $\mathrm{O}-\mathrm{Na}$ anticorrelation is evident from our UVES data, in agreement with past results.

This analysis shows the power of FLAMES for analysis of globular clusters: the accurate reddenings and metal abundances obtained by a procedure like that described here, combined with distance determinations from cluster dynamics or main sequence fitting, and high quality colour-magnitude diagrams, could allow derivation of ages with errors below 1 Gyr for individual globular clusters.
\end{abstract}

Key words. stars: abundances - stars: evolution - stars: Population II - Galaxy: globular clusters: general - Galaxy: formation

\section{Introduction}

Accurate estimates of the absolute ages of (the oldest) Globular Clusters (GCs), coupled with determinations of the Hubble constants $H_{0}$ from the spectrum of fluctuations of the microwave background determined from either the WMAP experiment (Spergel et al. 2003), or from the HST Key Project (Freedman et al. 2001) may provide a stringent lower limit to the age of the Universe and constrains the exponent $w$ of the equation of state for dark energy (Jimenez et al. 2003), independently of type Ia SN observations (see e.g. Gratton et al. 2003a).

^ Based on data collected at the European Southern Observatory with the VLT-UT2, Paranal, Chile (ESO 073.D-0100).

$\star \star$ Tables 3, 4 and 7 are only available in electronic form at the CDS via anonymous ftp to cdsarc. u-strasbg. fr

(130.79.128.5) or via

http://cdsweb.u-strasbg.fr/cgi-bin/qcat?J/A+A/440/901
Furthermore, within the framework of a standard $\Lambda \mathrm{CDM}$ model, where the age of the Universe is accurately fixed at $13.7 \pm 0.2$ Gyr by the WMAP results (Spergel et al. 2003), the age of GCs can be used to constrain the epoch of formation of the Galaxy, linking the local Universe to the distant one (see Carretta et al. 2000; Gratton et al. 2003a).

Relative ages are fundamental to describing the early history of our Galaxy. In this framework it should be noted that galactic GCs divide into two main groups: halo and thick disk (or bulge) GCs (Zinn 1985). The differential ages method suggests that these two groups might have ages that differ by about 2 Gyr (Rosenberg et al. 1999), a result that seems supported by absolute ages (Gratton et al. 2003a). This has implications for both cosmology, where only the oldest GCs are of interest, and galactic evolution. Observations of GC systems in other galaxies suggest a link between GC formation and strong dynamical interactions (Peebles \& Dicke 1968; Schweizer \& Seitzer 1993). The oldest group of GCs might then be related to the very early phases of the galactic collapse, while the second 
one may instead trace a later accretion event (see Freeman \& Bland-Hawthorn 2002), possibly related to the end of the thick disk phase indicated by chemistry (Gratton et al. 1996; Gratton et al. 2000; Fuhrmann 1998).

An important goal is then to derive absolute ages with internal errors of \pm 1 Gyr for a sample of GCs. Ages for GCs with such small errors may be derived using only the luminosity of the turn-off (TO), which in turn requires accurate distances, with errors $<5 \%$. In perspective, most accurate and robust distances (error $<2 \%$ ) for a few GCs will be obtained using geometrical methods (Piotto et al. 2004). At present, distances with errors of 3-5\% can be obtained for a larger sample of GCs using the Main Sequence Fitting Method (MSFM), exploiting local subdwarfs as standard candles (see Gratton et al. 1997; Pont et al. 1998; Carretta et al. 2000; Gratton et al. 2003a). Main sources of errors in MSFM are possible systematic differences in reddenings and metallicities between field and GC stars, while accurate initial He abundances in GCs have been determined by Cassisi et al. (2003; see also Salaris et al. 2004). Both of them can be reduced to within the required accuracy if a reddening-free temperature indicator is used for both field and GC stars of similar evolutionary phases. Analysis of the results of the ESO LP 165.L-0263 (Gratton et al. 2003a) relative to three GCs (NGC 6397, NGC 6752 and 47 Tuc), spanning almost the total metallicity range of galactic GCs, showed that this approach may provide reddenings accurate to $\Delta(B-V)= \pm 0.005 \mathrm{mag}$, metallicities accurate to $\pm 0.04 \mathrm{dex}$, distances accurate to $4 \%$, and ages with errors of about \pm 1 Gyr. Also, geometrical distances, which determines the true distance modulus toward a cluster, will take advantage of accurate reddening and metallicity determinations, since apparent distance moduli are required to derive ages.

Note that here we are only interested in relative reddening and metallicity determinations: the adopted scale may be tied to that of field stars exploiting the clusters observed within the LP 165.L-0263 (47 Tuc, NGC 6397, and NGC 6752: Gratton et al. 2003a). The same temperature indicator may be adopted (H $\alpha$ profile).

In this paper we describe a pilot program on NGC 6752 , which exploits the multiplexing capabilities of FLAMES, the VLT multifibre facility (Pasquini et al. 2002). The large number of spectra that could be obtained using GIRAFFE allowed proper reduction of the major source of errors in temperatures derived from $\mathrm{H} \alpha$ : flat fielding. On the other hand, low resolution and $S / N$ were not too critical in such observations, allowing use of faint turn-off stars. The simultaneous acquisition of spectra of a few red giants with UVES additionally allowed accurate determination of the chemical composition. For this purpose we preferred to use relatively warm stars, for which the analysis is expected to be robust. This procedure's success suggests the usefulness of an extensive program on other globular clusters. Ages within \pm 1 Gyr are now fully within reach for a substantial sample in both Zinn's groups.

\section{Observations}

Data used in this paper are based on a single $1300 \mathrm{~s}$ exposure obtained on June 24th, 2004, with FLAMES at
Table 1. Summary of observations.

\begin{tabular}{lc}
\hline \hline Date & $24 / 06 / 2004$ \\
Time (UT Start) & $09: 49: 51$ \\
Exposure Time (s) & 1300 \\
Airmass (Mean) & 1.803 \\
Seeing FWHM (arcsec) & 0.62 \\
\hline
\end{tabular}

Kueyen (=VLT2) in service mode. The observations were obtained at a rather large airmass (about 1.8 ) and in very good seeing conditions ( $F W H M=00^{\prime} 62$ at zenith and $5000 \AA$ ) (see also Table 1).

120 fibres feeding the GIRAFFE spectrograph were centred on stars slightly brighter than the turn-off of NGC 6752 in the magnitude range $16.7<V<17.2$. Stars were carefully selected from high quality photometric $U B V$ observations obtained with the Wide-Field Imager (WFI) at the $2.2 \mathrm{~m}$ ESO-MPI telescope (total field of view of $34 \times 33 \mathrm{arcmin}^{2}$ ). For a detailed representation of the data reduction and calibration of this data set we refer the reader to Momany et al. (2004).

The astrometric calibration of NGC 6752 reference images employed over 7000 stars from the GSC2.2 catalogue (Loomis et al. 2004) using the IRAF MSCRED package ${ }^{1}$. The internal accuracy of the astrometry has been estimated to be about 0.15 arcsec, well within the FLAMES requirements $(0.2$ arcsec). To further confirm fulfilment of the requirements, we matched our astrometrically calibrated NGC 6752 catalogue with that of UCAC2 (Zacharias et al. 2004) and estimated the positional residuals for 550 stars in common. The residuals show a Gaussian distribution with an $\mathrm{rms}$ of $\simeq 0.05 \mathrm{arcsec}$ in both coordinates.

Only uncrowded stars were considered, that is, stars not showing any companion brighter than $V_{\text {target }}+2$ mag within 2.5 arcsec, or brighter than $V_{\text {target }}-2$ mag within 10 arcsec. The targets were selected to lie close to the cluster mean loci in the colour-magnitude diagram. A posteriori, radial velocities confirmed membership of all but two of the observed stars. Eight fibres were additionally used to monitor sky background; they were pointed toward carefully selected empty sky regions.

The GIRAFFE spectrograph was used with the LR06 grating; the spectra cover the wavelength range 6400-7100 $\AA$ at a resolution of about $R \sim 6000$. Pixel-to-pixel $S / N$ of the spectra (measured from the scatter of individual spectral points in the wavelength range $6660-6670 \AA$ ) ranged from 5 to 50 , with typical values around 20 . The $S / N$ values were generally lower for stars in the outer regions of the cluster, most likely because these stars were not well centred on the fibre heads. This can be attributed to the effects of differential refraction at the rather large airmass of observation.

The average of the eight sky spectra were used to subtract telluric emissions (in particular, emission in $\mathrm{H} \alpha$ ) from

${ }^{1}$ IRAF is distributed by the National Optical Astronomy Observatory, which is operated by the Association of Universities for Research in Astronomy, Inc, under cooperative agreement with the National Science Foundation. 
GIRAFFE spectra. Appropriate scaling factors were evaluated to take into account the transmission of individual fibres.

Seven fibres feeding the UVES spectrograph were centred on stars close to the RGB bump $(13.2<V<14.2)$, while one was dedicated to the sky. The spectra cover the wavelength range 4700-6900 $\AA$ and have $20<S / N<40$.

The two sets of spectra were reduced using the dedicated FLAMES pipelines (BLDRS Python software 0.5.3 version for the GIRAFFE spectra; uves/2.1.1 version for UVES spectra). We found that this UVES pipeline does not accurately subtract the background between orders in the green-yellow part of the spectra. Only a few lines measured on these portions of the spectra were considered in the present analysis.

\section{Reddening estimates from GIRAFFE spectra}

\subsection{Fluxes}

The following procedure was used to derive accurate temperatures from the $\mathrm{H} \alpha$ profile. First, instrumental fluxes within 10 narrow bands of $5 \AA$ width in the region including $\mathrm{H} \alpha$ were measured on the GIRAFFE spectra by integrating the observed spectra, after shifting them in wavelength for the geocentric radial velocity of each star. Cosmic ray hits were removed before evaluating the fluxes. The list of the bands used is given in Table 2.

The fluxes measured in each band were then normalized to a pseudocontinuum given by a straight line connecting the average of the first two bands with the average of the two last bands. The normalized fluxes for all the stars observed with GIRAFFE are given in Table 3 (available only in electronic form at the CDS). The second column of this table gives also the $S / N$ ratio for each spectrum, computed from the spectral region $6660-6670 \AA$, where there is no significant feature.

Since the $\mathrm{H} \alpha$ profiles are expected to be fairly symmetric and since bands are defined symmetrically with respect to the line center, errors in these normalized fluxes can be obtained by comparing fluxes measured on the blue and red side of the $\mathrm{H} \alpha$. Eliminating a few outliers, the comparisons are as follows:

$5-6=-0.008 \pm 0.002, \quad \mathrm{rms}=0.019, \quad 112$ stars

and:

$4-7=-0.013 \pm 0.002, \quad \mathrm{rms}=0.020, \quad 114$ stars

for bands 5-6 and 4-7. From these comparisons, we expect a typical error on the average of $\langle 5,6\rangle$ bands of \pm 0.0095 , corresponding to an internal error in $T_{\text {eff's }}$ of $\pm 110 \mathrm{~K}$ (see next section). This error agrees with expectations based on the $S / N$ of the spectra.

Individual heliocentric radial velocities measured by the FLAMES pipeline are presented in the third column of Table 3. In a few cases ( 7 stars out of 120), these measures of the radial velocities by the automatic routine in the pipeline were obviously wrong, perhaps due to the strong telluric signal present in this wavelength range. Radial velocities for these stars were measured by fitting $\mathrm{H} \alpha$, and zeroing the radial velocity on the telluric bands consistently with the other stars. All but one (29049) of the stars appear to be members of the cluster
Table 2. Definition of the bands used to derive $T_{\text {eff }}$ 's.

\begin{tabular}{lcc}
\hline \hline Band & $\begin{array}{c}\text { Start } \\
(\AA)\end{array}$ & $\begin{array}{c}\text { End } \\
(\AA)\end{array}$ \\
\hline 1 & 6537.8 & 6542.8 \\
2 & 6542.8 & 6547.8 \\
3 & 6547.8 & 6552.8 \\
4 & 6552.8 & 6557.8 \\
5 & 6557.8 & 6562.8 \\
6 & 6562.8 & 6567.8 \\
7 & 6567.8 & 6572.8 \\
8 & 6572.8 & 6577.8 \\
9 & 6577.8 & 6582.8 \\
10 & 6582.8 & 6587.8 \\
\hline
\end{tabular}

on the basis of radial velocity; the velocity for star 39462 is discrepant, too, but this spectrum has very low $S / N$, so we suspend judgement about it. However we did exclude these two stars from our estimates of reddening of NGC 6752. The mean radial velocity is $-32.0 \pm 0.6 \mathrm{~km} \mathrm{~s}^{-1}$, with an rms scatter of individual values of $6.0 \mathrm{~km} \mathrm{~s}^{-1}$ (118 stars), with no obvious correlation of the spread with neither $S / N$ nor distance from the cluster centre.

The average value of the radial velocity agrees very well with those estimated by Webbink (1988: $-32.2 \pm 3.2 \mathrm{~km} \mathrm{~s}^{-1}$ ) and Dubath et al. (1997: $\left.-32.0 \pm 1.6 \mathrm{~km} \mathrm{~s}^{-1}\right)$, while it is slightly larger than that given by Rutledge et al. (1997: $-27.4 \pm$ $2.7 \mathrm{~km} \mathrm{~s}^{-1}$ ). For comparison, the seven stars observed with UVES provided a slightly lower average velocity $(-23.8 \pm$ $2.1 \mathrm{~km} \mathrm{~s}^{-1}$, see Table 6).

Errors in the GIRAFFE radial velocities should be roughly $5-6 \mathrm{~km} \mathrm{~s}^{-1}$, as given by the FLAMES pipeline. For comparison, the radial velocity error is expected to be roughly $\sigma(R V) \sim$ $33 /(S / N) \mathrm{km} \mathrm{s}^{-1}$ when using the formula by Landman et al. (1982) and assuming that the radial velocity signal is given by $\mathrm{H} \alpha$ alone. For the typical $S / N$ of the program spectra, errors are then expected in the range $0.7-6 \mathrm{~km} \mathrm{~s}^{-1}$. Given these large uncertainties on the errors attached to these radial velocities, they are of little use in estimating the internal velocity dispersion in NGC 6752.

\subsection{Effective temperatures}

Effective temperatures were derived by comparing the normalized average fluxes in bands 5 and 6 with those expected from Kurucz (1992) model atmospheres (with the overshooting option switched off) of different temperatures. Gravities and metal abundances adopted for these model atmospheres are those appropriate for the program stars. The $\mathrm{H} \alpha$ absorption and broadening were modelled using the same assumptions as Castelli et al. (1997). The theoretical profiles were further broadened by convolution with a Gaussian profile mimicking the instrumental profile.

To put these effective temperature derivations in a clearer perspective, we show in Fig. 1 the average spectrum for the stars with Giraffe spectra with $S / N>25$ in the region 


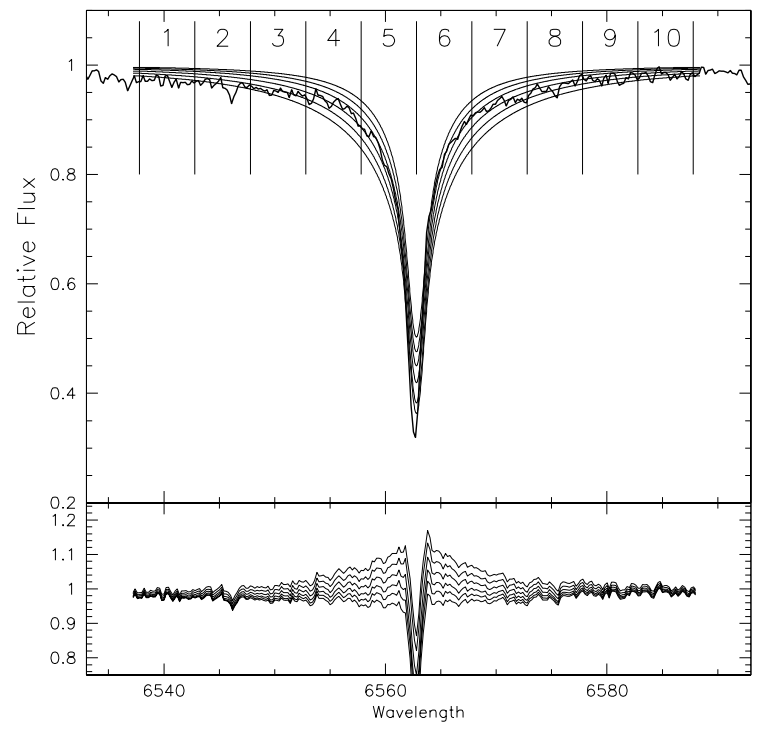

Fig. 1. Top panel: average $\mathrm{H} \alpha$ spectrum for the stars with Giraffe spectra with $S / N>25$. Superimposed are synthetic spectra computed for gravities and metal abundances appropriate for the program stars and various values of $T_{\text {eff }}(=5600,5800,6000,6200,6400,6600 \mathrm{~K})$. The limits of the bands used are also shown as vertical marks. Bottom panel: ratios between observed and synthetic spectra.

around $\mathrm{H} \alpha$. Superimposed are synthetic spectra computed for gravities and metal abundances appropriate for the program stars and various values of $T_{\text {eff }}(=5600,5800,6000,6200$, $6400,6600 \mathrm{~K})$. The limits of the bands used are also shown as vertical marks.

Values for the $T_{\text {eff's }}$ are given in Table 4 (available only in electronic form at the CDS).

\section{Photometry and reddening}

Reddening estimates for individual stars can be derived by comparing the observed colours with those predicted from the reddening-free temperatures and an appropriate colour-temperature relation.

The $B-V$ colours were given by the WFI photometry (Momany et al. 2004). They were corrected blueward by $0.020 \mathrm{mag}$ to put them on the same scale of Thompson et al. (1999) used in Gratton et al. (2003a). Based on the scatter around the mean relation, we expect that errors in colours for individual stars are of $\pm 0.025 \mathrm{mag}$ at this magnitude. We

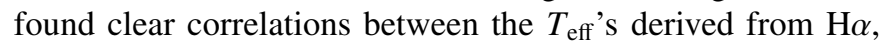
the $B-V$ colours, and the $V$ magnitudes within our sample (see Fig. 2).

Reddenings toward NGC 6752 can finally be evaluated by comparing the observed colour-temperature relation with that expected from models. Each individual star provided a reddening estimate, as listed in the last column of Table 4. No clear trend in these reddening estimates with e.g. $V$ magnitude or location on the field could be discerned. The individual values were then averaged together to provide a best estimate. Values obtained in this way are listed in Table 5. For comparison, the value obtained by using a similar procedure from spectra of 20 stars taken at higher resolution (Gratton et al. 2003a) is also

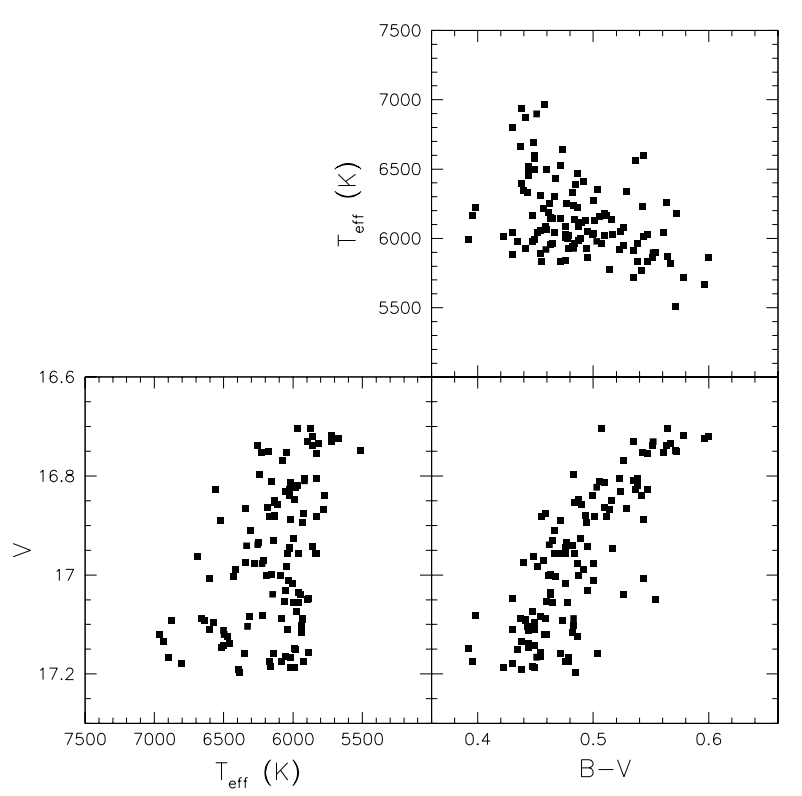

Fig. 2. Top panel: effective Temperature $-B-V$ colour diagram for the stars observed with GIRAFFE; Left panel: effective Temperature $V$ magnitude diagram for the same stars; right panel: $B-V$ colour $V$ magnitude diagram for the same stars.

Table 5. Various reddening estimates.

\begin{tabular}{lcc}
\hline \hline Estimates & $\begin{array}{c}E(B-V) \\
\mathrm{mag}\end{array}$ & $\begin{array}{c}\mathrm{rms} \\
\mathrm{mag}\end{array}$ \\
\hline All stars (118) & $0.046 \pm 0.005$ & 0.053 \\
Only stars with $S / N>15(90)$ & $0.042 \pm 0.005$ & 0.049 \\
& & \\
Gratton et al. (2003a) & $0.040 \pm 0.005$ & \\
Harris (1996) & 0.04 & \\
Schlegel et al. (1998) & 0.056 & \\
\hline
\end{tabular}

given, as well as the values from the compilation by Harris (1996) and from the reddening maps of Schlegel et al. (1998).

There are several aspects in this procedure that may introduce systematic errors in our reddening derivations: systematic uncertainties in flat fielding, inappropriate modelling of $\mathrm{H} \alpha$, errors in the model atmospheres, photometric errors etc. However, we recall here that what matters for the age derivations are not the absolute values of the reddenings, but instead the relative values between cluster and field stars. The good agreement with the determination of Gratton et al. (2003a), which is on a scale consistent with that adopted for the field subdwarfs, supports the present technique.

\section{Metallicity from UVES spectra}

\subsection{Equivalent widths}

Table 6 gives the main parameters for the stars observed with UVES. The colours have been corrected blueward by 0.008 mag to put them onto the same scale of Thompson et al. (1999). Note that this correction is slightly different from that 
Table 6. Photometry and spectrum data for stars observed with UVES.

\begin{tabular}{cccccc}
\hline \hline Star & $V$ & $B-V$ & $\begin{array}{c}R V \\
\mathrm{~km} \mathrm{~s}^{-1}\end{array}$ & $S / N$ & $\begin{array}{c}\sigma(E W) \\
(\mathrm{m} \AA)\end{array}$ \\
\hline 25072 & 14.199 & 0.803 & -26.4 & 28 & 3.8 \\
26059 & 13.444 & 0.853 & -17.7 & 40 & 2.6 \\
30409 & 13.568 & 0.840 & -22.9 & 44 & 2.7 \\
30426 & 14.075 & 0.802 & -30.0 & 28 & 3.1 \\
34854 & 13.698 & 0.814 & -15.5 & 19 & 5.8 \\
37999 & 13.273 & 0.866 & -25.3 & 32 & 2.9 \\
39672 & 13.662 & 0.825 & -29.1 & 23 & 10.6 \\
\hline
\end{tabular}

found appropriate for the TO-stars, which suggests the presence of a colour term in one of the two photometry.

The $E W \mathrm{~s}$ were measured on the spectra using the ROSA code (Gratton 1988; see Table 7) with Gaussian fittings to the measured profiles, which exploit a linear relation between $E W \mathrm{~s}$ and $F W H M$ of the lines, derived from a subset of lines characterized by cleaner profiles. Since the observed stars span a very limited parameter range, errors in these $E W$ s can be computed by comparing values derived from individual stars with the average value for the whole sample. Typical errors obtained using this procedure are listed in the last column of Table 6 . They are roughly reproduced by the formula $\sigma(E W) \sim 100 /(S / N) \mathrm{m} \AA$. Considering the resolution and sampling of the spectra, the errors in the $E W \mathrm{~s}$ are in agreement with expectations based on photon noise statistics (Cayrel 1988). Finally, we notice that due to the problems in background subtraction in the greenyellow part of the spectra, only lines with wavelength $>5900 \AA$ were considered, save for $\mathrm{Na}, \mathrm{Mg}$, and $\mathrm{Si}$, for which also lines in the 5600-5750 ̊ region were considered.

\subsection{Atmospheric parameters}

Effective temperatures were derived from dereddened $B-V$ colour using the calibration by Alonso et al. (1999): we interpolated the values at $[\mathrm{Fe} / \mathrm{H}]=-1.5$ from the tables for $[\mathrm{Fe} / \mathrm{H}]=-1$ and -2 . Rather than directly using the individual stellar colours, we preferred to use the colours of the mean loci at the same magnitudes of the program stars. Individual stellar colours would have produced larger individual errors ( $0.014 \mathrm{mag}$, corresponding to $\pm 34 \mathrm{~K}$, rather than $< \pm 10 \mathrm{~K}$ with the procedure adopted here: see below).

Internal uncertainties in these temperatures can be obtained by considering the errors in the $V$ magnitudes $(<0.05 \mathrm{mag})$ and the slope of the magnitude temperature relationship, which is $193 \mathrm{~K} / \mathrm{mag}$ in the range of interest for the program stars. We get internal uncertainties of $<10 \mathrm{~K}$, corresponding to about $0.011 \mathrm{dex}$ in $[\mathrm{Fe} / \mathrm{H}]$. Systematic errors are larger. The uncertainty in the reddening $( \pm 0.005 \mathrm{mag})$ multiplied for the slope of the colour-temperature relation (about $-2400 \mathrm{~K} / \mathrm{mag}$ ) yields a systematic error of $\pm 12 \mathrm{~K}$, which is about $0.013 \mathrm{dex}$ in $[\mathrm{Fe} / \mathrm{H}]$. Much larger is the uncertainty in the adopted temperature scale, most likely in the range $50-100 \mathrm{~K}$, producing possible errors in the Fe abundances in the range 0.055-0.11 dex.
Table 8. Atmospheric parameters for stars observed with UVES.

\begin{tabular}{lcccc}
\hline \hline Star & $T_{\text {eff }}$ & $\log g$ & {$[\mathrm{~A} / \mathrm{H}]$} & $v_{\mathrm{t}}$ \\
\hline 25072 & 5033 & 2.48 & -1.49 & 1.50 \\
26529 & 4888 & 2.12 & -1.49 & 1.50 \\
30409 & 4911 & 2.17 & -1.49 & 1.40 \\
30426 & 5009 & 2.42 & -1.49 & 1.30 \\
34854 & 4937 & 2.24 & -1.49 & 1.45 \\
37999 & 4854 & 2.02 & -1.49 & 1.50 \\
39672 & 4929 & 2.22 & -1.49 & 1.45 \\
\hline
\end{tabular}

Table 9. Iron abundances for stars observed with UVES.

\begin{tabular}{lcccccc}
\hline \hline Star & \multicolumn{3}{c}{$\mathrm{Fe} \mathrm{I}$} & & & $\mathrm{Fe}$ II \\
& $n$ & {$[\mathrm{Fe} / \mathrm{H}]$} & $\mathrm{rms}$ & $n$ & {$[\mathrm{Fe} / \mathrm{H}]$} & $\mathrm{rms}$ \\
\hline 25072 & 26 & -1.46 & 0.10 & 1 & -1.59 & \\
26529 & 26 & -1.50 & 0.08 & 3 & -1.53 & 0.05 \\
30409 & 25 & -1.51 & 0.10 & 2 & -1.59 & 0.09 \\
30426 & 25 & -1.46 & 0.11 & 2 & -1.51 & 0.04 \\
34854 & 23 & -1.54 & 0.15 & 1 & -1.56 & \\
37999 & 31 & -1.45 & 0.08 & 3 & -1.53 & 0.05 \\
39672 & 16 & -1.43 & 0.24 & & & \\
\hline$\langle[\mathrm{Fe} / \mathrm{H}] \mathrm{I}\rangle=-1.48 \pm 0.01$ & & & & \\
$\langle[\mathrm{Fe} / \mathrm{H}] \mathrm{II}\rangle=-1.55 \pm 0.02$ & & & & \\
\hline
\end{tabular}

Surface gravities were obtained from the location of the stars in the colour-magnitude diagram. This procedure requires assumptions about the distance modulus (from Gratton et al. 2003a), the bolometric corrections (from Alonso et al. 1999), and about the masses (we assumed a mass of $0.9 M_{\odot}$, close to the value given by isochrones fittings). Uncertainties in these gravities are small; we estimate a total error of about $0.15 \mathrm{dex}$, dominated by systematic effects in the temperature scale.

Microturbulent velocities $v_{\mathrm{t}}$ were determined by eliminating trends in the relation between expected line strength and abundances (see Magain 1984). To estimate errors in these values we notice that we found that the error in the $E W$ s contributes $57 \%$ of the variance of the errors in the abundances for individual lines. Given the typical uncertainties in the slope of expected line strength vs. abundances, this implies an expected random error in the microturbulent velocities of $\pm 0.09 \mathrm{~km} \mathrm{~s}^{-1}$. This value coincides with the star-to-star scatter in microturbulent velocities.

Finally, model metal abundances were set in agreement with derived Fe abundance. The adopted model atmosphere parameters are listed in Table 8.

\subsection{Fe abundances}

Individual $[\mathrm{Fe} / \mathrm{H}]$ values are listed in Table 9, as well as averages over the whole sample. Reference solar abundances are as in Gratton et al. (2003b). The average Fe abundance from all stars is $[\mathrm{Fe} / \mathrm{H}]=-1.48 \pm 0.02$ (error of the mean) with an rms scatter of 0.038 dex from 7 stars. If we consider only 
Table 10. Uncertainties in Fe abundances for stars observed with UVES.

\begin{tabular}{|c|c|c|c|c|c|c|c|}
\hline Parameter & Variation & {$[\mathrm{Fe} / \mathrm{H}] \mathrm{I}$} & {$[\mathrm{Fe} / \mathrm{H}] \mathrm{II}$} & $\begin{array}{c}\text { Random } \\
\text { Error }\end{array}$ & $\begin{array}{c}\text { Systematic } \\
\text { Error }\end{array}$ & $\begin{array}{c}\text { Total } \\
\text { Random }\end{array}$ & $\begin{array}{l}\text { Total } \\
\text { Syst. }\end{array}$ \\
\hline$E W \mathrm{~s}$ & & & & & & 0.013 & 0.006 \\
\hline $\log g f$ & & & & & & & 0.019 \\
\hline$T_{\text {eff }}$ & $100 \mathrm{~K}$ & 0.109 & -0.025 & $10 \mathrm{~K}$ & $50 \mathrm{~K}$ & 0.011 & 0.054 \\
\hline $\log g$ & $+0.3 \mathrm{dex}$ & -0.013 & 0.125 & $0.02 \mathrm{dex}$ & $0.15 \mathrm{dex}$ & 0.000 & 0.006 \\
\hline$[\mathrm{A} / \mathrm{H}]$ & $+0.2 \mathrm{dex}$ & 0.003 & -0.013 & 0.03 dex & 0.06 dex & 0.001 & 0.001 \\
\hline$v_{\mathrm{t}}$ & $+0.2 \mathrm{~km} \mathrm{~s}^{-1}$ & -0.045 & -0.025 & $0.09 \mathrm{~km} \mathrm{~s}^{-1}$ & $0.06 \mathrm{~km} \mathrm{~s}^{-1}$ & 0.020 & 0.013 \\
\hline Total & & & & & & 0.026 & 0.056 \\
\hline
\end{tabular}

the five stars with $S / N>25$, we have: $[\mathrm{Fe} / \mathrm{H}]=-1.48 \pm 0.01$ with an rms scatter of 0.028 dex.

There is a small offset of 0.07 dex between abundances given by neutral and singly ionized Fe I lines. This might be attributed to the use of very few lines for Fe II, but might also indicate some errors $(\sim 0.15 \mathrm{dex})$ in the surface gravities, as well as a systematic error of about $50 \mathrm{~K}$ in the effective temperatures. For comparison, Gratton et al. (2001) found $[\mathrm{Fe} / \mathrm{H}]=$ -1.44 for neutral iron, and -1.55 for singly ionized iron.

Table 10 lists the impact of various uncertainties on the derived Fe abundances. Variations in parameters of the model atmospheres (effective temperatures $T_{\text {eff }}$, surface gravities $\log g$, model metal abundances $[\mathrm{A} / \mathrm{H}]$, microturbulent velocities $v_{\mathrm{t}}$ ) were obtained by changing each of the parameters at a time. The second column gives the variation of the parameter used to estimate the changes in the abundances from neutral (Col. 3) and singly ionized (Col. 4) Fe lines. Columns 5 and 6 give the random (i.e. appropriate to each star) and systematic (scale errors for all stars) uncertainties in the various parameters, while Cols. 7 and 8 give the corresponding errors in the Fe abundances. The last row gives total errors, which have been obtained by combining errors due to the various parameters.

\subsection{Intrinsic star-to-star scatter in Fe abundances}

The observed star-to-star scatter in Fe abundances is very small, in particular if only higher quality $(S / N>25)$ spectra are considered. In spite of this, one may wonder if there is some evidence for real star-to-star scatter in the Fe abundances, or at least put some upper limit on this scatter, even though the sample of stars observed with UVES is not extensive. In Sect. 5.3 we have seen that the expected star-to-star scatter in Fe abundances due to the adopted temperatures is only $0.008 \mathrm{dex}$. More relevant is the error due to the microturbulent velocities.

To evaluate this source of error, we first note that considering only spectra with $S / N>25$, the error in abundances from individual lines from each spectrum is 0.068 dex, while the line-to-line rms scatter of the average abundances from the 5 spectra is 0.097 dex. This indicates that only part of the variance in the internal abundance errors is due to random errors in the $E W \mathrm{~s}$, variable from star-to-star. The remaining contribution can be attributed to systematic errors proper to each line (oscillator strengths, blends and systematic effects on positioning of the continuum level). Given these facts, the typical internal error in the abundances due to $E W \mathrm{~s}$ can be estimated to be $0.068 / \sqrt{26}=0.013 \mathrm{dex}$. Also, in the same way we may distribute the measured errors in the microturbulent velocities (determined from the $1-\sigma$ uncertainty in the slope of the expected line strength vs. abundance fit) between random (i.e. star-to-star variable) and systematic (i.e. constant throughout the analysis of all stars) errors. Only the random (star-to-star variable) error should be considered when discussing the star-to-star abundance variations. By combining the various sources of random errors quadratically, we get a prediction of 0.026 dex for the star-to-star spread in the Fe abundances. This compares very well with the measured star-to-star scatter of $0.028 \mathrm{dex}$. The conclusion is that there is very scarce evidence for an intrinsic star-to-star scatter in the abundances; a one-sided 1- $\sigma$ upper limit is 0.017 dex.

\subsection{O-Na anticorrelation}

The O-Na anticorrelation in NGC 6752 is well known from previous observations of both TO and subgiant stars (Gratton et al. 2001; Carretta et al. 2005), as well as of red giants (Carretta 1994; Norris \& Da Costa 1995; Yong et al. 2003). It is fully confirmed by the present data for RGB bump stars (see Fig. 3). Figure 3 also collects all data available up to now, which clearly shows how an extensive O-Na anticorrelation can be seen along all evolutionary phases.

\subsection{Abundance of other elements}

Table 11 lists the average abundances obtained for various elements. Also in this case, solar abundances were as in Gratton et al. (2003a). In the same table we also compare the abundances obtained in this paper with the analysis of Gratton et al. (2003a) and James et al. (2004). The comparison is very good for the best determined elements: we found clear overabundances of the $\alpha$-elements $([\alpha / \mathrm{Fe}]=+0.27 \pm 0.01)$, a small deficiency of $\mathrm{Ni}$, and slight overabundance of Ba. We notice that NGC 6752 closely traces the composition of the dissipative component considered by Gratton et al. (2003b), in agreement with its kinematics (Dinescu et al. 1999). The overabundance of $\alpha$-elements looks quite similar to those of other globular clusters (see e.g. Gratton et al. 2004). 
Table 11. Element-to-element abundance ratios for individual stars, averaged over the whole sample. For comparison, we also give the values found by Gratton et al. (2003a) and James et al. (2004).

\begin{tabular}{|c|c|c|c|c|c|c|c|c|c|c|}
\hline Star & $n$ & {$[\mathrm{O} / \mathrm{Fe}] \mathrm{I}$} & $n$ & {$[\mathrm{Na} / \mathrm{Fe}] \mathrm{I}$} & $n$ & {$[\mathrm{Mg} / \mathrm{Fe}] \mathrm{I}$} & $n$ & {$[\mathrm{Si} / \mathrm{Fe}] \mathrm{I}$} & $n$ & {$[\mathrm{Ca} / \mathrm{Fe}] \mathrm{I}$} \\
\hline 25072 & 1 & -0.09 & 2 & +0.35 & 2 & +0.41 & 3 & +0.34 & 9 & +0.29 \\
\hline 26529 & 1 & -0.10 & 2 & +0.39 & 2 & +0.41 & 2 & +0.18 & 9 & +0.37 \\
\hline 30409 & 1 & +0.46 & 2 & +0.18 & 2 & +0.60 & 3 & +0.37 & 10 & +0.43 \\
\hline 30426 & 1 & +0.18 & 2 & +0.27 & 2 & +0.41 & 3 & +0.22 & 10 & +0.38 \\
\hline 34854 & 1 & +0.33 & 2 & +0.31 & 2 & +0.69 & 3 & +0.45 & 7 & +0.43 \\
\hline 37999 & 1 & +0.59 & 1 & -0.01 & 2 & +0.52 & 4 & +0.32 & 12 & +0.34 \\
\hline 39672 & 1 & $<-0.07$ & 1 & +0.46 & 2 & +0.52 & 1 & +0.31 & 6 & +0.30 \\
\hline Average & & +0.19 & & +0.29 & & +0.51 & & +0.31 & & +0.36 \\
\hline error & & 0.11 & & 0.06 & & 0.04 & & 0.03 & & 0.02 \\
\hline $\mathrm{rms}$ & & 0.28 & & 0.16 & & 0.11 & & 0.09 & & 0.06 \\
\hline compare to & & & & & & +0.28 & & & & +0.31 \\
\hline Star & $n$ & {$[\mathrm{Sc} / \mathrm{Fe}] \mathrm{II}$} & $n$ & {$[\mathrm{Ti} / \mathrm{Fe}] \mathrm{I}$} & $n$ & {$[\mathrm{Ni} / \mathrm{Fe}] \mathrm{I}$} & $n$ & {$[\mathrm{Ba} / \mathrm{Fe}] \mathrm{II}$} & {$[\alpha / \mathrm{Fe}]$} & \\
\hline 25072 & 1 & +0.26 & 1 & +0.30 & 2 & -0.15 & 2 & +0.27 & +0.34 & \\
\hline 26529 & 1 & -0.04 & 1 & +0.19 & 2 & -0.14 & 2 & +0.29 & +0.29 & \\
\hline 30409 & 1 & -0.01 & 2 & +0.15 & 2 & -0.11 & 2 & +0.39 & +0.39 & \\
\hline 30426 & & & 2 & +0.17 & 2 & -0.18 & 2 & +0.45 & +0.30 & \\
\hline 34854 & 1 & +0.05 & & & 2 & -0.21 & 2 & +0.37 & +0.44 & \\
\hline 37999 & 2 & -0.04 & 2 & +0.16 & 2 & -0.25 & 2 & +0.28 & +0.34 & \\
\hline 39672 & & & & & 1 & -0.32 & 2 & +0.43 & +0.33 & \\
\hline Average & & +0.06 & & +0.19 & & -0.19 & & +0.35 & +0.35 & \\
\hline error & & 0.05 & & 0.03 & & 0.03 & & 0.03 & 0.02 & \\
\hline $\mathrm{rms}$ & & 0.12 & & 0.06 & & 0.07 & & 0.07 & 0.05 & \\
\hline compare to & & -0.06 & & +0.20 & & -0.11 & & +0.18 & +0.27 & \\
\hline
\end{tabular}

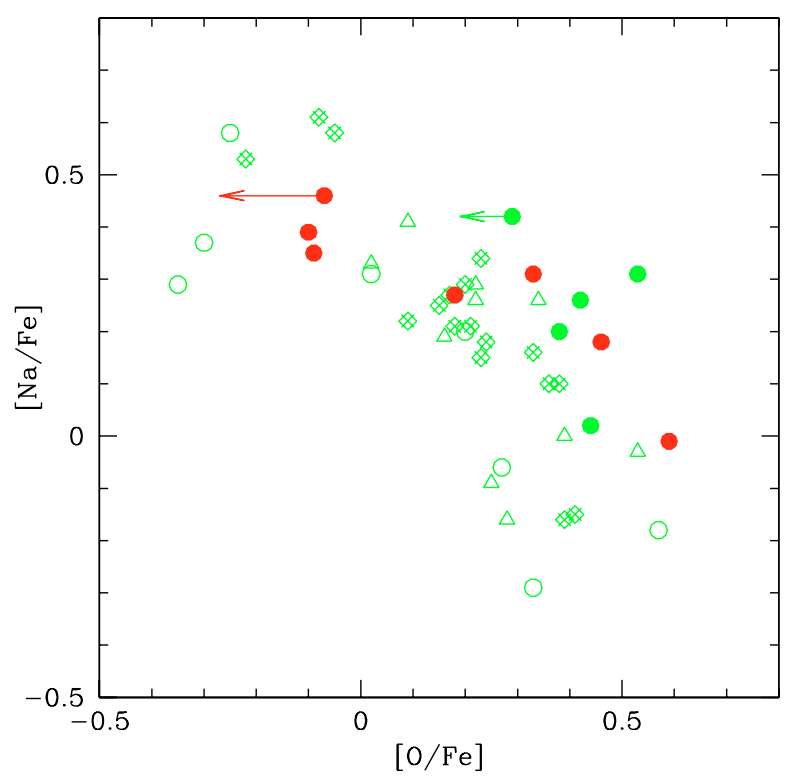

Fig. 3. $[\mathrm{Na} / \mathrm{Fe}]$ ratio as a function of $[\mathrm{O} / \mathrm{Fe}]$ for stars in NGC 6752. Red filled circles are our RGB bump stars in the present study. Green-filled and open circles are subgiant and turn-off stars, respectively, from Carretta et al. (2005). Literature data are as follow: green diamonds with crosses inside are bright red giants from the extensive study by Yong et al. (2003), and open green triangles are red giant stars from Norris \& Da Costa (1995; 6 stars) and Carretta (1994; 4 stars).

\section{Conclusions}

We have shown that a single $1300 \mathrm{~s}$ exposure with FLAMES at VLT2 can provide accurate estimates of the reddening toward NGC 6752, as well as of the chemical composition of the cluster (errors of $0.005 \mathrm{mag}$ and of $0.02 \mathrm{dex}$, respectively). Similar analyses may provide results of comparable accuracy for other globular clusters, too, on a uniform scale ${ }^{2}$. While results of similar accuracy have already been obtained for a few clusters (including NGC 6752), use of FLAMES allows such high accuracy with much less observing time (about a factor of 20). An extensive program over a large number of clusters may reduce errors in age determination for those clusters for which accurate distances could be obtained from either the main sequence fitting method, or even better from dynamical methods.

Acknowledgements. This research was funded by PRIN 2003029437 "Continuità e discontinuità nella formazione della nostra Galassia" by the Italian MIUR.

\section{References}

Alonso, A., Arribas, S., \& Martinez-Roger, C. 1999, A\&AS, 140, 261 Carretta, E. 1994, Ph.D. Thesis, Un. Padua

Carretta, E., Gratton, R. G., Clementini, G., \& Fusi Pecci, F. 2000, ApJ, 533, 215

Carretta, E., Gratton, R. G., Lucatello, S., Bragaglia, A., \& Bonifacio, P. 2005, A\&A, 433, 597

Cassisi, S., Salaris, M., \& Irwin, A. W. 2003, ApJ, 588, 862

Castelli, F., Gratton, R. G., \& Kurucz, R. L. 1997, A\&A, 318, 841

${ }^{2}$ Of course, scale errors would be much larger. However, as mentioned in the Introduction, this is not a serious concern if reddening and abundance scale are tied to those of globular clusters by an appropriate consistent analysis as in Gratton et al. (2003a). 
Cayrel, R. 1988, in The Impact of Very High $S / N$ Spectroscopy on Stellar Physics, ed. G. Cayrel de Strobel, \& M. Spite (Dordrecht: Kluwer Academic Publishers), IAU Symp., 132, 345

Dinescu, D. L., Girard, T. M., \& van Altena, W. F. 1999, AJ, 117, 1792

Dubath, P., Meylan, G., \& Mayor, M. 1997, A\&A, 324, 505

Freedman, W. L., Madore, B. F., Gibson, B. K., et al. 2001, ApJ, 553, 47

Freeman, K., \& Bland-Hawthorn, J. 2002, ARA\&A, 40, 487

Fuhrmann, K. 1998, A\&A, 338, 161

Gratton, R. G. 1988, Rome Obs. Preprint, 29

Gratton, R. G., Carretta, E., Matteucci, F., \& Sneden, C. 1996, in Formation of the Galactic Halo... Inside and Out, ed. H. Morrison, \& A. Sarajedini, ASP Conf. Ser., 92, 307

Gratton, R. G., Fusi Pecci, F., Carretta, E., et al. 1997, ApJ, 491, 749

Gratton, R. G., Carretta, E., Matteucci, F., \& Sneden, C. 2000, A\&A, 358,671

Gratton, R. G., Bonifacio, P., Bragaglia, A., et al. 2001, A\&A, 369, 87

Gratton, R. G., Bragaglia, A., Carretta, E., et al. 2003a, A\&A, 408, 529

Gratton, R. G., Carretta, E., Claudi, R., Lucatello, S., \& Barbieri, M. 2003b, A\&A, 408, 529

Gratton, R. G., Sneden, C., \& Carretta, E. 2004, ARA\&A, 42, 385

Harris, W. E. 1996, AJ, 112, 1487

James, G., François, P. Bonifacio, P., et al. 2004, A\&A, 414, 1071

Jimenez, R., Verde, L., Treu, T., \& Stern, D. 2003, ApJ, 593, 622

Kurucz 1992, in The Stellar Populations of Galaxies, ed. B. Barbuy, \& A. Renzini (Dordrecht: Kluwer Academic Publishers), IAU Symp., 149, 225

Landman, D. A., Roussel-Duprè, R., \& Tanigawa, G. 1982, ApJ, 261, 732
Loomis, C. G., McLean, B. J., Greene, G. R., \& White, R. L. 2004, BAAS, 205, 9110

Magain, P. 1984, A\&A, 134, 189

Momany, Y., Bedin, L. R., Cassisi, S., et al. 2004, A\&A, 420, 605

Norris, J., \& Da Costa, G. S. 1995, ApJ, 441, L81

Pasquini, L., Avila, G., Blecha, A., et al. 2002, The Messenger, 110, 1

Peebles, P. J. E., \& Dicke, R. H. 1968, ApJ, 154, 891

Piotto, G., Bedin, L. R., Cassisi, S., et al. 2004, Mem. SAIt Suppl., 5, 71

Pont, F., Mayor, M., Turon, C., \& Vandenberg, D. A. 1998, A\&A, 329, 87

Rosenberg, A., Saviane, I., Piotto, G., \& Aparicio, A. 1999, AJ, 118, 2306

Rutledge, G. A., Hesser, J. E., Stetson, P. B., et al. 1997, PASP, 109, 883

Salaris, M., Riello, M., Cassis, S., \& Piotto, G. 2004, A\&A, 420, 911

Schlegel, D. J., Finkbeiner, D. P., \& Davis, M. 1998, ApJ, 500, 525

Schweizer, F., \& Seitzer, P. 1993, ApJ, 417, L29

Spergel, D. N., Verde, L., Peiris, H. V., et al. 2003, ApJS, 148, 175

Thompson, I. B., Kaluzny, J., Pych, W., \& Krzeminski, W. 1999, AJ, 118,462

Webbink, R. F. 1988, in The Harlow-Shapley Symposium on Globular Cluster Systems in Galaxies (Dordrecht: Kluwer Ascademic Press), IAU Symp., 126, 49

Yong, D., Grundahl, F., Lambert, D. L., Nissen, P. E., \& Shetrone, M. D. 2003, A\&A, 402, 985

Zacharias, N., Urban, S., Zacharias, M., et al. 2004, AJ, 127, 3043

Zinn, R. 1985, ApJ, 293, 424 